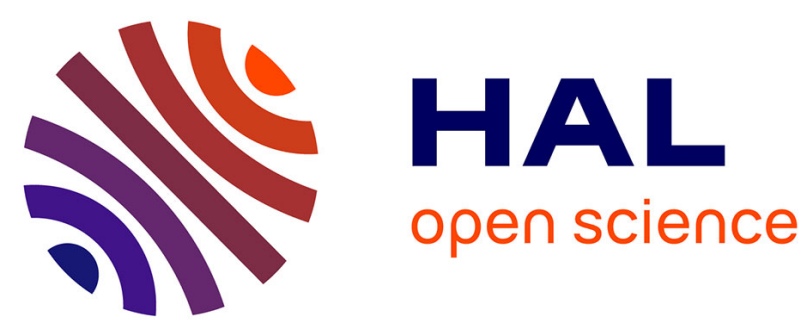

\title{
Strong Ifitm1 Expression In Cd4 T Cells In Hiv Controllers Is Correlated With Immune Activation
}

Etienne Canoui, Nicolas Noël, Camille Lécuroux, Faroudy Boufassa, Asier

Sáez-Cirión, Christine Bourgeois, Olivier Lambotte, Anrs Co21 Codex Study

Group

\section{To cite this version:}

Etienne Canoui, Nicolas Noël, Camille Lécuroux, Faroudy Boufassa, Asier Sáez-Cirión, et al.. Strong Ifitm1 Expression In Cd4 T Cells In Hiv Controllers Is Correlated With Immune Activation. Journal of Acquired Immune Deficiency Syndromes - JAIDS, 2017, 10.1097/QAI.0000000000001166 . pasteur01420412

\section{HAL Id: pasteur-01420412}

\section{https://hal-pasteur.archives-ouvertes.fr/pasteur-01420412}

Submitted on 16 May 2017

HAL is a multi-disciplinary open access archive for the deposit and dissemination of scientific research documents, whether they are published or not. The documents may come from teaching and research institutions in France or abroad, or from public or private research centers.
L'archive ouverte pluridisciplinaire HAL, est destinée au dépôt et à la diffusion de documents scientifiques de niveau recherche, publiés ou non, émanant des établissements d'enseignement et de recherche français ou étrangers, des laboratoires publics ou privés.

\section{(1)(1) $\$(0)$}

Distributed under a Creative Commons Attribution - NonCommercial - ShareAlikel 4.0 
1 Strong ifitm1 expression in CD4 T cells in HIV controllers is correlated with

\section{2 immune activation}

3 Etienne Canoui ${ }^{1,}{ }^{*}$, Nicolas Noël ${ }^{1,2,3,4}{ }^{*}$, Camille Lécuroux ${ }^{1,4}$, Faroudy Boufassa ${ }^{2,5,6}$, Asier 4 Sáez-Cirión ${ }^{7}$, Christine Bourgeois ${ }^{1,2,4}$, Olivier Lambotte ${ }^{1,2,3,4}$ and the ANRS CO21 CODEX 5 study group

6

7 1: INSERM U1184, Immunologie des infections virales et autoimmunité, Le Kremlin-Bicêtre, France

$8 \quad$ 2: Université Paris Sud, Le Kremlin-Bicêtre, France

9 3: Assistance Publique - Hôpitaux de Paris, Service de Médecine Interne et Immunologie Clinique,

10 Groupe Hospitalier Universitaire Paris Sud, Hôpital Bicêtre, Le Kremlin-Bicêtre, France

${ }^{4}$ : CEA, DSV/iMETI, Division of Immuno-Virology, IDMIT, France

5: INSERM U1018, Centre de recherche en Epidémiologie et Santé des Populations, Le KremlinBicêtre, France

6: Assistance Publique - Hôpitaux de Paris, Département d'épidémiologie, Groupe Hospitalier Universitaire Paris Sud, Hôpital Bicêtre, Le Kremlin-Bicêtre, France

7: Institut Pasteur, Unité HIV inflammation et persistance, Paris, France

*: co-first authors, equal contribution

\section{$\S:$ Corresponding author}

Pr Olivier Lambotte, Service de Médecine Interne et Immunologie Clinique

CHU Bicêtre ; 78, rue du Général Leclerc, 94275 Le Kremlin-Bicêtre CEDEX, FRANCE

Phone: +33 145212 783, Fax: +33 145212733

olivier.lambotte@aphp.fr

Count: Main document 1451 words, 1 figure, 20 references, 1 supplemental figure, 1 supplemental table

Running head: ifitm1 expression in HIV controllers 
1 Key words: Type 1 Interferon, Interferon stimulated genes, IFITM1, HIV controllers, 2 HIV.

3 


\section{INTRODUCTION}

2 During HIV infection, the Interferon (IFN)-stimulated genes (ISGs) have been associated with HIV pathophysiology by restricting HIV replication [1], or enhancing the immune activation [2,3]. The expression levels of ISGs depend on their function, as well as their regulation mechanisms and the viral progression $[4,5]$. Indeed, most ISGs are upregulated in CD4 and CD8 T cells from viremic untreated patients [5-7], and tend to diminish on antiretroviral therapy (ART). However, whereas monocytes are important in HIV pathogenesis [8], few studies have focused on ISGs regulation in monocytes from HIV-infected patients [9].

Little is known regarding the expression of ISGs in the rare patients who spontaneously control HIV replication (HIV controller patients (HICs)). In PBMCs from HICs, ifit1 and mxA seem overexpressed as compared with HDs and ART treated patients [10]. We have recently showed that plasma IP10 levels are higher in HICS than HDs and associated with low CD4 T cell counts [11]. Other studies reported that some ISGs known to restrict HIV, such as samhd1, schlaffen 11, apobec3c and apobec3d might be overexpressed in CD4 T cells or PBMCs from HICs [12-14].

Here, we studied the expression of four ISGs in three sorted cell subtypes (CD4 and CD8 T lymphocytes, and monocytes). We compared their expressions between HICs, HIV-1 infected ART-treated (ARTs), viremic treatment-naïve patients (VIRs), and healthy donors (HD). The expression of these ISGs according to the levels of T cell- and monocyte activation was analyzed. A strong ifitm1 expression in CD4 T cells in HIV controllers was correlated with immune activation. 


\section{PATIENTS AND METHODS}

2 HICs $(n=11)$ were enrolled in the ANRS CO21 CODEX cohort [11] with the following

3 definition: known HIV diagnosis for at least 5 years prior to enrolment, with HIV VL < 4400 copies $/ \mathrm{mL}$ in the last five consecutive measurements. ART-treated patients $(n=8)$

5 and VIRs ( $n=8)$ were defined as previously reported [11]. HIV - negative healthy donors (HDs) $(n=8)$ were recruited as controls. All patients gave their informed consent for this study, in accordance with the Helsinki declaration.

Freshly collected PBMCs were stained with the following conjugated monoclonal antibodies: PE-CD14, PerCP Cy5.5-CD8, APC-CD3, and APC-H7-CD4, before the FACS-based sorting procedure on BD ARIA (BD Biosciences ${ }^{T M}$ ). CD4+ T cells, CD8+ $T$ cells and monocytes (CD3-CD14+) were sorted and immediately lysed in RNeasy Lysis Buffer (QIAGEN*) and cryopreserved at $-80^{\circ} \mathrm{C}$. RNA isolation and reverse transcription were performed according to the manufacturers' protocols (RNeasy mini kit, Qiagen ${ }^{T M}$, and enhanced avian RT-PCR Kit, SIGMA ${ }^{T M}$, respectively). qRT-PCR analysis was performed using a SyBR Green RT PCR method (LightCycler system, Roche ${ }^{T M}$ ). All results were normalized for gapdh expression.

Levels of soluble CD14 (sCD14) were determined by ELISA (R\&D Systems, Minneapolis, Minnesota, USA), and the surface expression of HLA-DR and CD38 on T cells was analyzed by flow cytometry, as described previously [11].

For statistical analyses, continuous variables were compared using the nonparametric Kruskall-Wallis test followed by Dunn's test for multiple analysis. Categorical variables were compared using chi ${ }^{2}$ test, and Spearman's coefficient was used for correlation analyses. The threshold for statistical significance was set to $p$ 
1 less than 0.05. Data were stored and analysed using PRISM software (version 5,

2 GraphPad software, La Jolla, California, USA).

3 


\section{RESULTS}

The characteristics of the study population are summarized in the Supplemental

Table 1. There was no difference in terms of age and gender between each group of patients.

\section{Ifitm1 expression is elevated in CD4 T cells from HICs}

The analysis of ISGs expression in the 4 groups and the 3 cell subtypes is depicted in Figure 1. In VIRs, the expression of ifitm1 in monocytes, pkr in all cell types, $m x A$ in CD4 and CD8 T cells, and ifit1 in CD4 were higher than in ARTs, suggesting a strong role of the virus in ISGs induction. Similar results were seen when comparing ISGs expression between VIRs and HICs, except for $m x A$ in all cell types and ifit1 in CD8 T cells and monocytes, for which the expression were similar in the two groups. Importantly, we found that the expression of ifitm1 expression in CD4 T cells from HICs $(p=0.01)$ and ARTs $(p=0.001)$ was similar to VIRs and higher than HDs. In contrast, the expression of ifitm1 in CD8 T cells and monocytes and those of the other genes in the three cell subtypes were similar in HICs and in HDs.

\section{The expression of most ISGs is correlated positively with HIV viral load}

In view of these observations, we hypothesized that the expression of most ISGs was associated with the HIV viral load (VL). Indeed, pooling the data of all HIV infected patients, the HIV VL was significantly correlated with the expression of $p k r$ in CD4 T cells, CD8 T cells, and monocytes, as well as $m x A$ in CD4 and CD8 T cells, and with ifit1 in CD4 T cells. Of note, similar results were obtained when classifying the HIV VL into categorical variables (i.e., detectable or undetectable). Also, when 
1 considering all HIV infected patients, the CD4 T cell count was negatively correlated

2 with the expression of $p k r$ in CD4 T cells and monocytes (with a strong trend for CD8

3 T cells), and ifit1 only in CD4 T cells, but not with $m x A$ expression.

4 Interestingly, the expression of ifitm1 was associated with the HIV VL in monocytes

5 only, but was negatively correlated with CD4 count in every cell subtypes. These results suggested that ifitm1 expression could depend on other conditions than the

7 amount of virus itself, especially in T cells.

9 ifitm1 expression is associated with immune activation

Thus, we lastly investigated the relationships between ISGs expression and immune activation. When all HIV-1 infected patients were considered, positive correlations were observed between ifitm 1 expression in each cellular subtype and the corresponding activation markers (i.e. $\%$ of HLA-DR+CD38+CD4+ T cells $(r=0.4, p=$ $0.03)$ and CD8+ $T$ cells $(r=0.4, p=0.04)$, and SCD14 for monocyte activation $(r=0.48$, $p=0.02)$ ) (Supplemental Figure 1a). Such relationships were not observed with the other ISGs, except for ifit1 in CD4 T cells. Moreover, the correlation between ifitm1 expression in CD4 $T$ cells and the frequency of circulating HLADR+CD38+ CD4+ T cells was still observed when HICs were analyzed separately $(r=0.8, p=0.003)$ (Supplemental Figure 1b). 


\section{DISCUSSION}

2 We selected 4 ISGs with distinct production mechanisms and reported their

3 expression in three cell subsets in four groups of individuals. $p k r$ and ifit1 expressions

4 depend on the engagement of type I IFN, but can be also directly stimulated by HIV RNA or particles $[15,16]$. $m x A$ seems to be directly correlated with type I IFN, with a strong link with HIV viral load [17]. Conversely, ifitm1 expression depends on type I, but also type II IFN, with strong links to the proinflammatory milieu [16]. Consequently, viremic patients had higher levels of $p k r$ in each cell subtypes, $m \times A$ in CD4 and CD8 T cells, and ifit1 in CD4 T cells than the other groups. The correlation between these ISGs and the HIV viral load support the role of viral particles in their production $[5-7,18]$.

The most striking point was the finding that in HICs' CD4 T cells, ifitm1 was overexpressed compared with HDs, whereas no differences were found between HICs and HD for the other ISGs. This observation extends previous results from gene expression profiling of CD4 T cells from HICs but had never been quantified by RT-PCR in sorted cells $[13,14]$. ifitm 1 has been recently described as part of a HIV-1 restriction factor family $[19,20]$. Moreover, it has been suggested that ifitm1 might be induced by IL-6 or other acute-phases cytokines [16]. This is consistent with our observations that ifitm1 was globally linked with immune activation but barely with the viral load, the correlation being still present only when HICs are studied.

It seems important to separately analyze the expression of ISGs in purified CD4 T cells and monocytes, since the role of monocytes in the pathophysiology of HIV and inflammation is increasing. The analysis of the ex vivo purified monocytic compartiment has seldom been reported in the field of ISG expression. Indeed, we observed that the expression profile of most ISGs was different between CD4 T cells 
1 and monocytes, suggesting that the individualization of these cell types might better

2 decipher the mechanisms of ISGs regulation in the setting of HIV infection.

3 Our results show that the expression profile of ISGs in HIV infected patients varies

4 according to the cell type, the disease status and the gene considered, with multiple

5 regulation mechanisms beyond type I/II Interferons. Importantly, the gene expression

6 profiles in monocytes should be distinguished from that in CD4 T cells. In HICs, ifitm1

7 seems to have a unique expression profile and is associated with immune CD4 T cell

8 activation. Its putative role in HIV pathogenesis and antiviral control deserves further 9 investigations. 


\section{REFERENCES}

1 Fenton-May AE, Dibben O, Emmerich T, Ding H, Pfafferott K, Aasa-Chapman MM, et al. Relative resistance of HIV-1 founder viruses to control by interferon-alpha. Retrovirology 2013; 10:146.

2 Fernandez S, Tanaskovic S, Helbig K, Rajasuriar R, Kramski M, Murray JM, et al. CD4+ Tcell deficiency in HIV patients responding to antiretroviral therapy is associated with increased expression of interferon-stimulated genes in CD4+ T cells. J Infect Dis 2011; 204:1927-1935.

3 Lederman MM, Calabrese L, Funderburg NT, Clagett B, Medvik K, Bonilla H, et al. Immunologic failure despite suppressive antiretroviral therapy is related to activation and turnover of memory CD4 cells. J Infect Dis 2011; 204:1217-1226.

4 Liu S-Y, Sanchez DJ, Aliyari R, Lu S, Cheng G. Systematic identification of type I and type II interferon-induced antiviral factors. Proc Natl Acad Sci U S A 2012; 109:4239-4244.

5 Hyrcza MD, Kovacs C, Loutfy M, Halpenny R, Heisler L, Yang S, et al. Distinct Transcriptional Profiles in Ex Vivo CD4+ and CD8+ T Cells Are Established Early in Human Immunodeficiency Virus Type 1 Infection and Are Characterized by a Chronic Interferon Response as Well as Extensive Transcriptional Changes in CD8+ T Cells. J Virol 2007; 81:3477-3486.

6 Sedaghat AR, German J, Teslovich TM, Cofrancesco J Jr, Jie CC, Talbot CC Jr, et al. Chronic CD4+ T-cell activation and depletion in human immunodeficiency virus type 1 infection: type I interferon-mediated disruption of T-cell dynamics. J Virol 2008; 82:1870-1883.

7 Rotger M, Dang KK, Fellay J, Heinzen EL, Feng S, Descombes P, et al. Genome-wide mRNA expression correlates of viral control in CD4+ T-cells from HIV-1-infected individuals. PLoS Pathog 2010; 6:e1000781.

8 Campbell JH, Hearps AC, Martin GE, Williams KC, Crowe SM. The importance of monocytes and macrophages in HIV pathogenesis, treatment, and cure. AIDS 2014;28:2175-87

9 Wu JQ, Sassé TR, Wolkenstein G, Conceicao V, Saksena MM, Soedjono M, et al. Transcriptome analysis of primary monocytes shows global down-regulation of genetic networks in HIV viremic patients versus long-term non-progressors. Virology 2013; 435:308-319.

10 Krishnan S, Wilson EMP, Sheikh V, Rupert A, Mendoza D, Yang J, et al. Evidence for innate immune system activation in HIV type 1-infected elite controllers. J Infect Dis 2014; 209:931-939.

11 Noel N, Boufassa F, Lécuroux C, Saez-Cirion A, Bourgeois C, Dunyach-Remy C, et al. Elevated IP10 levels are associated with immune activation and low $\mathrm{CD} 4{ }^{+}$T-cell counts in HIV controller patients. AIDS 2014; 28:467-476. 
12 Riveira-Muñoz E, Ruiz A, Pauls E, Permanyer M, Badia R, Mothe B, et al. Increased expression of SAMHD1 in a subset of HIV-1 elite controllers. J Antimicrob Chemother 2014; 69:3057-3060.

13 Abdel-Mohsen M, Raposo RAS, Deng X, Li M, Liegler T, Sinclair E, et al. Expression profile of host restriction factors in HIV-1 elite controllers. Retrovirology 2013; 10:106.

14 de Masson A, Kirilovsky A, Zoorob R, Avettand-Fenoel V, Morin V, Oudin A, et al. Blimp-1 overexpression is associated with low HIV-1 reservoir and transcription levels in central memory CD4+ T cells from elite controllers. AIDS 2014;28:1567-77

15 Clerzius G, Gélinas J-F, Gatignol A. Multiple levels of PKR inhibition during HIV-1 replication. Rev Med Virol 2011; 21:42-53.

16 Diamond MS, Farzan M. The broad-spectrum antiviral functions of IFIT and IFITM proteins. Nat Rev Immunol 2013; 13:46-57.

17 Haller O, Kochs G. Human MxA protein: an interferon-induced dynamin-like GTPase with broad antiviral activity. J Interferon Cytokine Res Off J Int Soc Interferon Cytokine Res 2011; 31:79-87.

18 Jacquelin B, Mayau V, Targat B, Liovat A-S, Kunkel D, Petitjean G, et al. Nonpathogenic SIV infection of African green monkeys induces a strong but rapidly controlled type IIFN response. J Clin Invest 2009; 119:3544-3555.

19 Lu J, Pan Q, Rong L, He W, Liu S-L, Liang C. The IFITM proteins inhibit HIV-1 infection. J Virol 2011; 85:2126-2137.

20 Compton AA, Bruel T, Porrot F, Mallet A, Sachse M, Euvrard M, et al. IFITM Proteins Incorporated into HIV-1 Virions Impair Viral Fusion and Spread. Cell Host Microbe 2014; 16:736-747. 
1 FIGURE LEGENDS

2

3 Fig. 1. Expression of ISGs between patient groups and cell types. (a) IFN-induced 4 transmembrane protein 1 (IFITM1). (b) RNA-activated Protein Kinase (PKR). (c) IFN-induced 5 protein with tetratricopeptide repeats (IFIT1). (d) Myxoma virus Resitance protein A (MxA). 6 HDs, healthy donors; HICs, HIV controllers; ARTs, aviremic ART-treated patients; VIRs, $7 \quad$ viremic treatment naïve patients.

8 


\section{FIGURE}

2 Figure 1.

A.

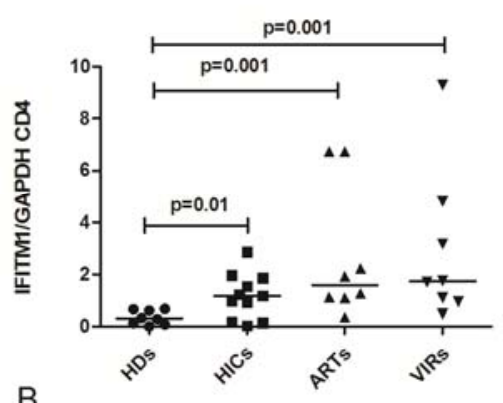

B.
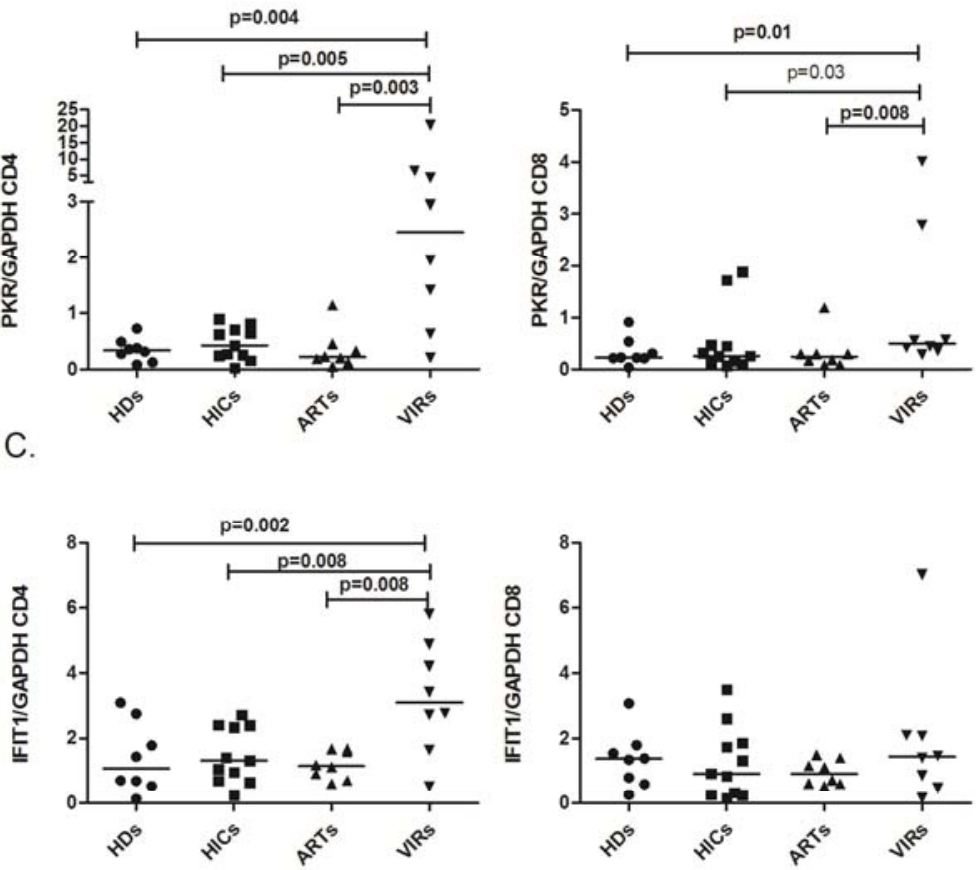

d)

3
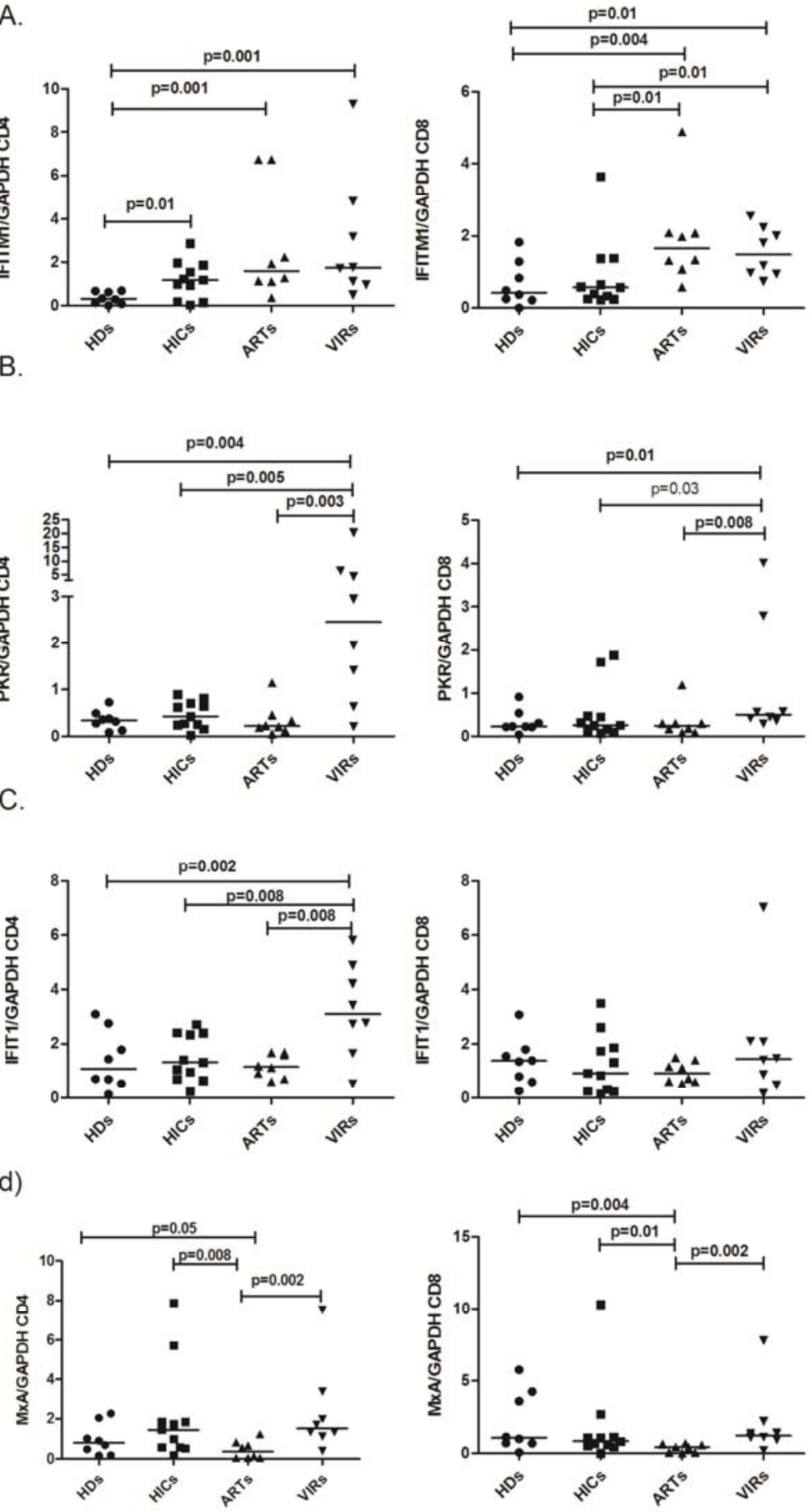
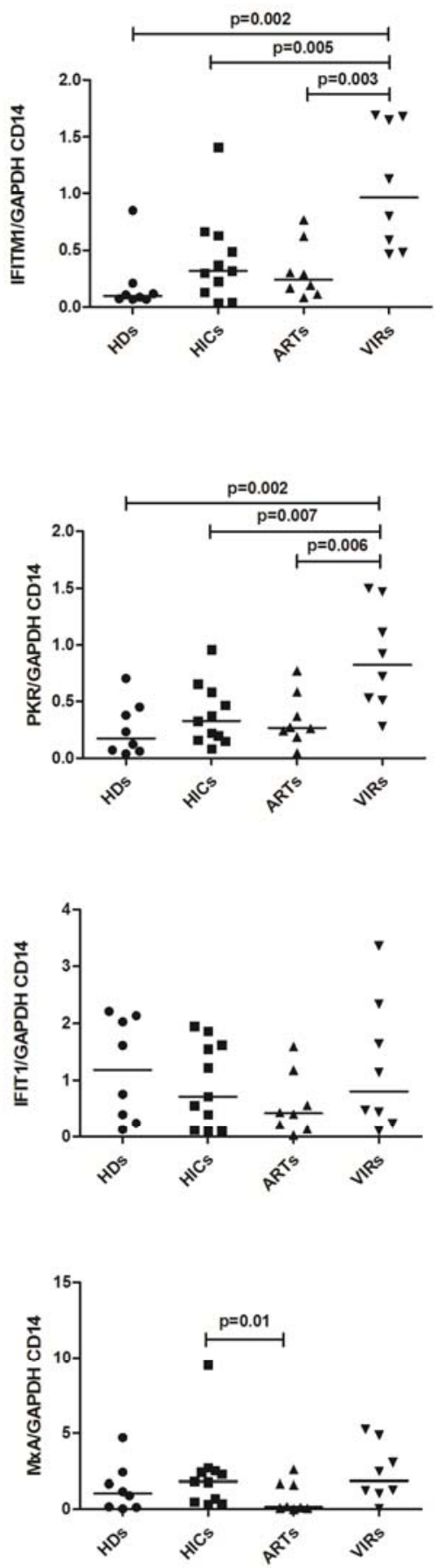
1 SUPPLEMENTAL MATERIAL

2 
1 FIGURE LEGEND

2 Supplemental Figure 1. Relationships with the ifitm1 expression and immune activation 3 marker (HLA-DR and CD38 for CD4 and CD8 T cells and level of SCD14 for the monocytes)

4 (a) Considering all HIV-1 infected patients. (b) Considering HICs separately. IFITM1, IFN5 induced transmembrane protein 1; HICs, HIV controllers; ARTs, aviremic ART-treated 6 patients; VIRs, viremic treatment naïve patients.

7 
Supplemental Figure 1

a)
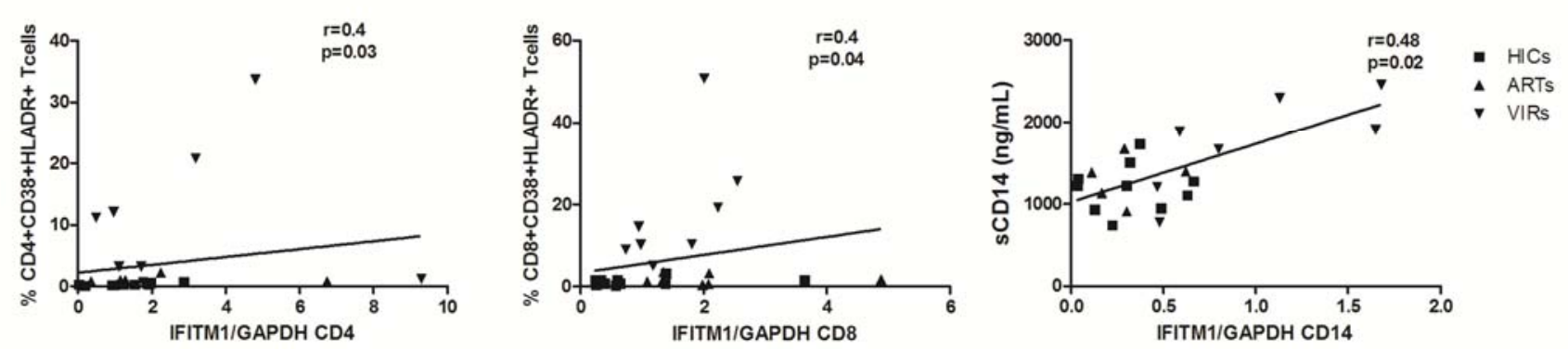

b)
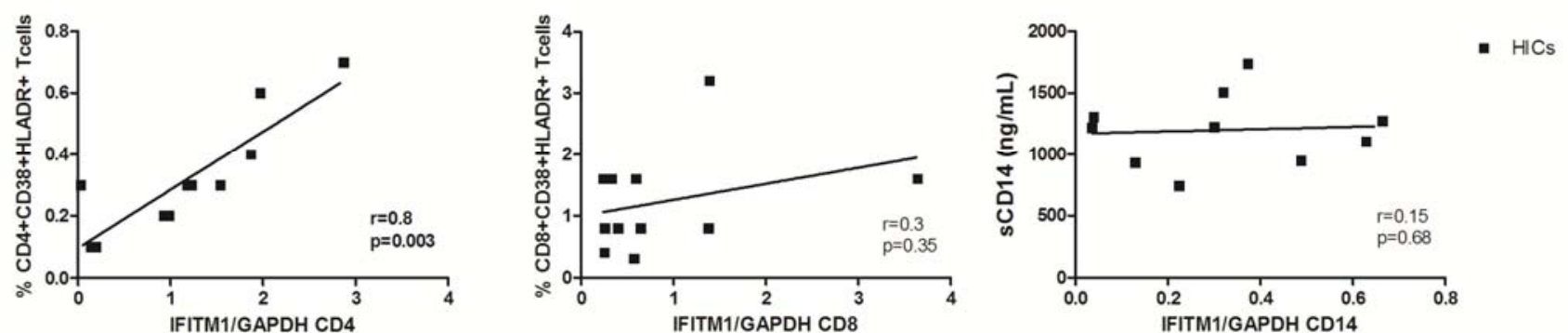
SUPPLEMENTAL FIGURESI TABLES

3

4 Supplemental Table 1. Characteristics of study participants 5 
Supplemental Table 1

Supplemental Table 1. Characteristics of study participants

\begin{tabular}{|c|c|c|c|c|c|}
\hline Characteristics & HDs $(n=8)$ & HICs $(n=11)$ & ARTs $(n=8)$ & VIRs $(n=8)$ & $p$ \\
\hline Age (years) & 46 [43.75-48.5] & $46.5[42.25-50.75]$ & 47 [43-53] & 37 [32-54] & 0.6 \\
\hline Male, $n(\%)$ & $3(37)$ & $4(36)$ & $4(50)$ & $3(37)$ & 0.7 \\
\hline $\begin{array}{l}\text { CD4+ T-cell count at enrollment } \\
\text { (cells } / \mu \mathrm{L})\end{array}$ & - & 875 [768-1001] & 467 [430-550] & $100[46-184]$ & $<0.01$ \\
\hline $\begin{array}{l}\text { Lowest CD4+ T-cell count since } \\
\text { HIV diagnosis (nadir) (cells/ } \mu \mathrm{L} \text { ) }\end{array}$ & - & 523 [464.5-637] & 285 [272-330] & $100[46-184]$ & $<0.01$ \\
\hline $\begin{array}{c}\text { Plasma HIV RNA VL at enrollment } \\
\text { (log10copies/ml) }\end{array}$ & - & $1.6[<1.6-1.6]$ & $<1.6[<1.6-<1.6]$ & $4.9[4.4-5.35]$ & $<0.01$ \\
\hline HCV coinfection, $n(\%)$ & $0(0)$ & $0(0)$ & $0(0)$ & $1(14)$ & 0.3 \\
\hline HBV coninfection, $n(\%)$ & $0(0)$ & $0(0)$ & $0(0)$ & $2(28)$ & 0.06 \\
\hline $\begin{array}{c}\text { Active opportunistic infection at } \\
\text { enrollment, } \mathrm{n}(\%)\end{array}$ & - & 0 & 0 & $5(62)$ & $<0.01$ \\
\hline
\end{tabular}

Results are shown as median (IQR) or $n(\%)$. HDs, healthy donors; HICs, HIV controllers; ARTs, aviremic ART-treated patients; VIRs, viremic treatment naïve patients ; VL, viral load 